\title{
ON AXISYMMETRIC DEFORMATIONS OF NONLINEAR VISCOELASTIC
} MEMBRANES

\section{ALAN WINEMAN}

Department of Applied Mechanics and Engineering Sciences, The University of Michigan, Ann Arbor, Michigan 48109 (U.S.A.)

(Received April 20, 1978)

\section{Summary}

In previous work it was shown that a class of axisymmetric viscoelastic membrane problems can be reduced to the numerical solution of a system of partial differential-Volterra integral equations. This general method has the disadvantage that a change in constitutive equation requires rederivation of specific details of the governing system. In this paper, a revised method is presented in which a change in constitutive equation does not affect the details of the governing equations. All that is needed is a change in the subprogram for computing stress from the deformation history.

The revised method is illustrated for the case of the inflation of a circular membrane by lateral pressure, a rheological experimental configuration. The membrane material is assumed to be a fluid such as polyisobutylene. This is in contrast to the solid type models used in all earlier examples. For this purpose, a BKZ model is developed from various sources.

\section{Introduction}

The study of the large deformation of thin polymer sheets is important in rheology and polymer forming. Such sheets can be modeled as viscoelastic membranes, for which a reasonably well developed theory exists in mechanics [1]. One very useful class of situations considers deformations which can be assumed axisymmetric. This includes two particular test configurations used in rheology for determining constitutive properties. In the first, an initially plane circular membrane is inflated by lateral pressure through a sequence of surfaces of revolution [2]. The polar region, which undergoes a locally homogeneous equal biaxial stretch history, is a source of data. The other test configuration 
utilizes an initially circular cylindrical tube, bonded at its ends to rigid plates [3]. Under combined inflation by internal pressure and elongation, the material at the midplane undergoes a locally homogeneous unequal biaxial stretch history. Within this class it is also possible to simulate drape or vacuum forming of polymer sheets. Although the deformation is axisymmetric, useful information can be gained with a minimum of complexity due to spatial variation.

Initial work on large axisymmetric deformations of nonlinear viscoelastic membranes is due to Wineman. Plane deformations of a membrane with a concentric hole were treated in [4], with the effects of spinning, included in [5]. The rheological problem of inflation by lateral pressure was presented in [6]. An essential theme in the above work is that these problems have a formulation leading to a system of equations which is particularly convenient for numerical solution. The general method is independent of the details of any particular integral type of constitutive equation. However, specific ones were used for numerical examples. An analytical constitutive equation embodying the essential features of a nonlinear viscoelastic solid was constructed for use in [4] and [5]. A constitutive equation developed experimentally for a styrene-butadiene rubber was used in [6].

In rheological applications, such as presented in [6], the problem may be solved for several different constitutive equations. These may represent different candidate models for a single material, in which case calculated deformed membrane profiles are to be compared to observed profiles. Alternatively, solutions could be obtained for different constitutive equations, representing different materials, in order to compare the membrane profiles. The same could be true for problems which model sheet processing. In the above referenced work, the general formulation is reduced to a system of three equations in certain kinematic variables. Among this system is a nonlinear partial differential-Volterra integral equation, which must be re-derived for each different choice of a constitutive equation. This necessitates spatial differentiation of the variables in the constitutive equation, substantial algebraic manipulation and computer modification.

This paper presents a modified approach which avoids these difficulties. In particular, an alternate reduction is given along with the associated modifications of the numerical procedure. It has the advantage that the governing field equations are unaffected by a change in the constitutive equation. The only change required is in the subprogram for computing stress from a given deformation history. This approach becomes especially useful in models whose time scale is affected by deformation, as in [7].

The reduction is based on a transformation which can be applied to any axisymmetric problem. However, it will be illustrated here in the context of the problem of the lateral inflation of an initially plane circular membrane. The general equations for this case are presented in Section 2 . The original reduction is outlined in Section 3 along with the numerical procedure. The proposed modification is discussed in Section 4 . A numerical example is given 
in Section 5. In all previous work in this area, the membrane material has been a viscoelastic solid. The numerical example here uses a model for a viscoelastic fluid.

\section{Formulation}

The formulation of the membrene inflation problem was discussed in detail in [6]. The main equations are summarized here.

The midsurface of the undeformed membrane is planar with a circular boundary of radius $a$. The initial thickness $h_{0}$ is uniform. A uniform time dependent pressure $p(t)$ is applied to one side of the membrane. Assuming quasistatic motion, the membrane remains clamped at its boundary while the midsurface deforms through a sequence of surfaces of revolution.

Let the polar axis of a cylindrical coordinate system coincide with the axes of symmetry of the deformed surfaces, and let the origin lie in the initial plane. A particle of the midsurface initially at $(r, \theta, 0)$ moves to $(\rho(r, t), \theta, z(r, t))$ at later time $t$ (see Fig. 1). In view of the axisymmetry, principal directions of stress and stretch at each time $t$ are known a priori to be fixed with respect to the material and tangent to the membrane in the meridional (1) and circumferential (2) directions and also normal to the surface. The principal stretch ratios in these directions are given by respectively,

$\lambda_{1}=\left[\left(\frac{\partial \rho}{\partial r}\right)^{2}+\left(\frac{\partial z}{\partial r}\right)^{2}\right]^{1 / 2}, \quad \lambda_{2}=\frac{\rho}{r}, \quad \lambda_{3}=\frac{1}{\lambda_{1} \lambda_{2}}$,

where $\lambda_{3}$ is determined by the incompressibility condition. If $\sigma_{\alpha}$ denotes a principal stress, its stress resultant per unit length of circumferential or meridional line in the membrane surface is $T_{\alpha}=h_{0} \lambda_{3} \sigma_{\alpha}(\alpha=1,2)$. At each time $t$, the force balance equations in the principal directions, transformed to

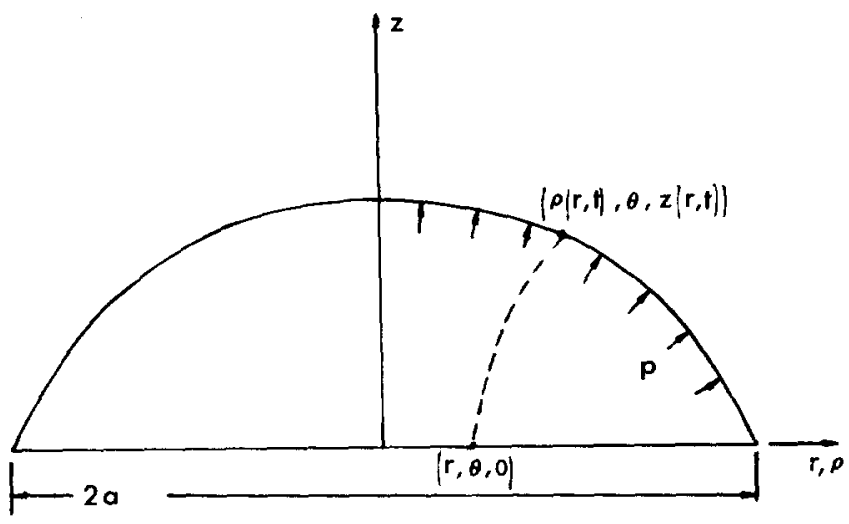

Fig. 1. Undeformed and deformed configurations of the inflated membrane. 
the initial configuration, are

$\frac{\partial T_{1}}{\partial r}+\frac{\eta\left(T_{1}-T_{2}\right)}{r \lambda_{2}}=0, \quad \eta=\frac{\partial \rho}{\partial r}$

$\kappa_{1} T_{1}+\kappa_{2} T_{2}=p$,

where principal curvatures $\kappa_{1}$ and $\kappa_{2}$ are given by

$\kappa_{1}=\frac{\eta \frac{\partial \lambda_{1}}{\partial r}-\lambda_{1} \frac{\partial \eta}{\partial r}}{\lambda_{1}^{2}\left[\lambda_{1}^{2}-\eta^{2}\right]^{1 / 2}}, \quad \kappa_{2}=\frac{\left[\lambda_{1}^{2}-\eta^{2}\right]^{1 / 2}}{r \lambda_{1} \lambda_{2}}$

As can be seen, the basic solution procedure can be carried out for all constitutive equations of integral type. For purposes of illustration, a nonlinear single integral type of constitutive equation is assumed. Let $\lambda(s)$ denote the pair $\left(\lambda_{1}(r, s), \lambda_{2}(r, s)\right)$. Then the model considered has the form

$\sigma_{\alpha}(t)=A_{\alpha}(\lambda(t), t)+\int_{0}^{t} B_{\alpha}[\lambda(t), \lambda(s), t-s] d s \quad(\alpha=1,2)$.

Functions $A_{\alpha}$ and $B_{\alpha}$ depend on the choice of material. Equation (2.4) encompasses the BKZ model [8] and finite linear viscoelasticity [9], whether solid or fluid. For viscoelastic fluids, the stress depends only on the relative strain history. Equation (2.4) can be regarded as a constitutive equation for a viscoelastic fluid if it is considered undeformed for $t<0$ and $B_{\alpha}$ has the form

$B_{\alpha}=\hat{B}_{\alpha}[\lambda(t) / \lambda(s), t-s]$.

For the problem and solution procedures considered here, and for the study of large axisymmetric deformations of viscoelastic membranes in general, it makes little difference whether the material is a solid or a fluid.

3. Reduction to a system for $\lambda_{1}, \lambda_{2}, \eta$

Equations (2.2)-(2.4) imply that the primary dependent variables are $\lambda_{1}$, $\lambda_{2}, \eta, \sigma_{1}$ and $\sigma_{2}$. Once these are known, $\rho(r, t)$ and $z(r, t)$ can be found from (2.1). The work in [6] reduces the problem to a system of equations for $\lambda_{1}$, $\lambda_{2}$ and the associated kinematic variable $\eta$.

The dominant equation is obtained by substituting (2.4) into the first of (2.2), which gives

$$
\begin{aligned}
& \frac{\partial \lambda_{1}}{\partial r}(t)\left\{F_{1}[\lambda(t), t]+\int_{0}^{t} G_{1}[\lambda(t), \lambda(s), t-s] d s\right\} \\
& \quad+\int_{0}^{t} \frac{\partial \lambda_{1}}{\partial r}(s) G_{2}[\lambda(t), \lambda(s), t-s] d s \\
& \quad=\frac{1}{r}\left\{F_{2}[\lambda(t), \eta(t), t]+\int_{0}^{t} G_{3}[\lambda(t), \lambda(s), \eta(s), t-s] d s\right\}
\end{aligned}
$$


The forms of $F_{1}, F_{2}, G_{1}, G_{2}, G_{3}$ in this partial differential-Volterra integral equation depend on the forms of $A_{\alpha}, B_{\alpha}$ in (2.4). The complexity of (3.1) results from the term $\partial T_{1} / \partial r$ in (2.2) and the complicated structure of $A_{\alpha}$ and $B_{\alpha}$. These depend on both the stretch tensor components and the stretch tensor invariants, which in turn depend on $\lambda(t)$ and $\lambda(s)$.

The operations of carrying out this differentiation to obtain expressions involving $\partial \lambda(t) / \partial r$ and $\partial \lambda(s) / \partial r$ and the subsequent algebra leading to (3.1) are very lengthy. The expression $\partial \lambda_{2} / \partial r$ which arises can be replaced so that the equation is entirely in terms of $\partial \lambda_{1} / \partial r$. This is done through the use of the compatibility relation obtained by eliminating $\rho$ between $\lambda_{2}$ and $\eta$ in (2.1) and (2.2):

$\partial \lambda_{2} / \partial r=\left(\eta-\lambda_{2}\right) / r$.

Forms for $F_{1}, F_{2}, G_{1}, G_{2}, G_{3}$ which arise in the case of a viscoelastic solid can be seen in the Appendix of [6]. When the material is a viscoelastic fluid, so that $B_{\alpha}$ depends on the ratio $\lambda(t) / \lambda(s)$ as in (2.5), these functions become even more complicated.

The system of equations for $\lambda_{1}, \lambda_{2}$ and $\eta$ is completed by substituting (2.3) into the second of (2.2) and rearranging:

$\frac{\partial \eta}{\partial r}=\frac{\eta}{\lambda_{1}} \frac{\partial \lambda_{1}}{\partial r}+\frac{\left[\lambda_{1}^{2}-\eta^{2}\right]}{r \lambda_{2}} \frac{\sigma_{2}}{\sigma_{1}}-\frac{p}{h_{0} \sigma_{1}} \lambda_{1}^{2} \lambda_{2}\left[\lambda_{1}^{2}-\eta^{2}\right]^{1 / 2}$.

Expressions for $\sigma_{1}$ and $\sigma_{2}$ are given by (2.4).

Boundary conditions are:

$r=0: \quad \lambda_{1}(0, t)=\lambda_{2}(0, t)=\eta(0, t)=A(t)$, say,

$r=a: \quad \lambda_{2}(a, t)=1$

The numerical procedure reduces the problem to the solution a system of three ordinary differential equations for $\lambda_{1}, \lambda_{2}$ and $\eta$ at a discrete set of times. If these times are $t_{1}=0, t_{2}, \ldots, t_{n-1}, t_{n}=t$, then the integrals can be approximated by finite sums of form

$\int_{0}^{t} G_{1}[\lambda(t), \lambda(s), t-s] \mathrm{d} s \simeq \sum_{k=1}^{n} W_{n k} G_{1}\left[\lambda\left(t_{n}\right), \lambda\left(t_{k}\right), t_{n}-t_{k}\right]$.

$W_{n k}$ are weighting functions associated with a particular approximation procedure and set of times. Suppose $\lambda_{1}\left(r, t_{k}\right), \lambda_{2}\left(r, t_{k}\right), \eta\left(r, t_{k}\right)$ and $\partial \lambda_{1}\left(r, t_{k}\right) / \partial r$, $k=1,2, \ldots, n-1$ have been found. Then (3.1) can be solved for $\partial \lambda_{1}\left(r, t_{n}\right) / \partial r$, which results in a nonlinear ordinary differential equation associated with time $t_{n}$ of form

$\frac{\partial \lambda_{1}}{\partial r}\left(r, t_{n}\right)=\Phi_{1}\left[\lambda_{1}\left(r, t_{n}\right), \lambda_{2}\left(r, t_{n}\right), \eta\left(r, t_{n}\right), r\right]$.

The dependence of $\Phi_{1}$ on $r$ arises from the presence of previously determined 
solutions in finite sums (3.5). Substituting (3.6) and (2.4) into (3.3) leads to an equation of form

$\frac{\partial \eta}{\partial r}\left(r, t_{n}\right)=\Phi_{2}\left[\lambda_{1}\left(r, t_{n}\right), \lambda_{2}\left(r, t_{n}\right), \eta\left(r, t_{n}\right), r\right]$.

Joining (3.2) to (3.6) and (3.7) leads to a system of ordinary differential equations for $\lambda_{1}, \lambda_{2}, \eta$ at time $t_{n}$ which can be integrated by standard numerical methods.

There are several numerical issues which arise in conjunction with the solution of this two-point boundary value problem. First, a method is needed for the determination of $A\left(t_{n}\right)$ at $r=0$ so that the boundary condition (3.4) at $r=a$ can be met. Second, as seen from (3.1), (3.2) and (3.3), the derivatives $\partial \lambda_{1} / \partial r, \partial \eta / \partial r$ and $\partial \lambda_{2} / \partial r$ are apparently indeterminate at $r=0$. Finally, it was pointed out in [6] that (3.3) can be replaced by

$\eta=\left[\lambda_{1}^{2}-\left(\frac{p \lambda_{1}^{2} \lambda_{2}^{2} r}{2 \sigma_{1}}\right)^{2}\right]^{1 / 2}$.

A detailed discussion of each of these issues is presented in [6], and will not be repeated here for the purpose of brevity.

\section{Revised reduction}

The revised reduction introduces the transformation

$\hat{\sigma}_{1}=\sigma_{1} / \lambda_{1}, \quad \hat{\sigma}_{2}=\sigma_{2} / \lambda_{2}, \quad \hat{\eta}=\eta / \lambda_{1}$.

Equations (2.2) and (3.2), using (2.3), become

$\frac{\partial \hat{\sigma}_{1}}{\partial r}=\frac{\hat{\sigma}_{2} \hat{\eta}-\hat{\sigma}_{1}}{r}$

$\frac{\partial \lambda_{2}}{\partial r}=\frac{\lambda_{1} \hat{\eta}-\lambda_{2}}{r}$

$\frac{\partial \hat{\eta}}{\partial r}=\frac{\left(1-\hat{\eta}^{2}\right)}{r} \frac{\hat{\sigma}_{2}}{\hat{\sigma}_{1}}-\frac{p}{h_{0}} \frac{\left(1-\hat{\eta}^{2}\right)^{1 / 2} \lambda_{1} \lambda_{2}}{\hat{\sigma}_{1}}$.

When (2.4) and (4.1) are combined and then joined with (4.2), the result is a system of three differential equations and two integral relations for $\lambda_{1}, \lambda_{2}$, $\hat{\eta}, \hat{\sigma}_{1}, \hat{\sigma}_{2}$ at time $t$. Proceeding formally, suppose $\lambda_{\alpha}(r, s)$ have been found for $0 \leqslant s<t$. Since these are known functions of $r,(2.4)$ and (4.1) can be regarded as implying

$\hat{\sigma}_{\alpha}(t)=S_{\alpha}\left(\lambda_{1}(t), \lambda_{2}(t), r, t\right) \quad(\alpha=1,2)$.

Since its derivative does not appear on the left hand side of (4.2), $\hat{\sigma}_{2}$ can be eliminated from (4.2) using (4.3). Finally, it is assumed that the expression for $\hat{\sigma}_{1}$ in (4.3) can be inverted to give the relation

$\lambda_{1}(t)=S^{*}\left(\hat{\sigma}_{1}(t), \lambda_{2}(t), r, t\right)$. 
This inversion is assumed possible when, relative to the principal directions, the constitutive equation expresses $\sigma_{\alpha}(t)$ explicitly in terms of $\lambda(t)$ as well as $\lambda(s), 0 \leqslant s \leqslant t$. This will be the case when the constitutive equation is of integral type, whether for solid or fluid behavior. The method of obtaining the inverse will be described shortly. The purpose of introducing this assumption of invertibility is to avoid the enormous effort required in the derivation of (3.1).

With (4.4), $\lambda_{1}(t)$ can be eliminated and (4.2) becomes a system of ordinary differential equations for $\hat{\sigma}_{1}, \hat{\eta}, \lambda_{2}$ at time $t$, i.e.

$$
\frac{\mathrm{d} \Lambda}{\mathrm{d} r}=\Phi(\Lambda, r), \quad \mathbf{\Lambda}=\left\{\begin{array}{l}
\hat{\sigma}_{1} \\
\hat{\eta} \\
\lambda_{2}
\end{array}\right\} .
$$

Revised boundary conditions are, by (3.4), (4.1) and (4.3):

$\hat{\eta}(0, t)=1, \quad \lambda_{2}(0, t)=A(t)$,

$\hat{\sigma}_{1}(0, t)=S_{1}(A(t), A(t), 0, t)$,

$\lambda_{2}(a, t)=1$.

As in Section 3, the numerical procedure used here determines the solution at a discrete set of times $t_{1}=0, t_{2}, \ldots, t_{n-1}, t_{n}=t$. The integrals in (2.4) are approximated by finite sums as in (3.5). Treating $\lambda_{1}\left(r, t_{k}\right)$ and $\lambda_{2}\left(r, t_{k}\right)$, $k=1,2, \ldots, n-1$, as known functions of $r$ in the approximations to (2.4), functions $S_{\alpha}$ in (4.3) become specific functions of $\lambda_{1}(t)$ and $\lambda_{2}(t)$. An explicit expression for the inverse function $S^{*}$ is not actually found. Instead, values for $S^{*}$ are found as needed. In particular, suppose system (4.5) is numerically integrated by; say, a fourth order Runge-Kutta method.

Let $\hat{\sigma}_{1}\left(r_{i}, t_{n}\right), \hat{\eta}\left(r_{i}, t_{n}\right), \lambda_{2}\left(r_{i}, t_{n}\right)$ and $\lambda_{1}\left(r_{i}, t_{n}\right)$ be known at space mesh point $r_{i}$. As part of this method, functions $\Phi$ in (4.5) are evaluated at these new arguments. Next, $\hat{\sigma}_{1}\left(r_{i}, t_{n}\right), \hat{\eta}\left(r_{i}, t_{n}\right)$ and $\lambda_{2}\left(r_{i}, t_{n}\right)$ are incremented using these values for $\Phi$. For this new set of values for $\hat{\sigma}_{1}$ and $\lambda_{2},(4.3)$ with $\alpha=1$ is a nonlinear equation for the new value of $\lambda_{1}$. This is solved by iteration, to yield the evaluated inverse $S^{*}$ at required arguments. With these new values for $\hat{\sigma}_{1}, \hat{\eta}, \lambda_{2}, \lambda_{1}$ and then $\hat{\sigma}_{2}$, the right hand side of (4.2) can be evaluated and the solution procedure continued. In actual practice, the converged value for $\lambda_{1}(t)$ during the previous inversion is used as the first estimate during the current iteration. Generally this is so close to the current value of $\lambda_{1}(t)$ that only a few iterations are needed.

In applying boundary conditions (4.6) at time $t_{n}$, the common value for $\lambda_{1}$ and $\lambda_{2}$ at $r=0, A\left(t_{n}\right)$, is assumed. $\hat{\sigma}_{1}(0, t)$ is then computed, as implied in (4.6). This avoids the inversion (4.4) at the first node.

It is seen that the present formulation leads to a modified system of equations and method of solution. However, the same numerical issues mentioned at the end of Section 3 also arise here. The only change is that (3.8) can be 
put in terms $\hat{\eta}$ and $\hat{\sigma}$, using (4.1). The previously mentioned discussion in [6] is still applicable.

In contrast with system (3.1), (3.2), (3.3), the revised system (4.2) has the same form for all constitutive equations. If a change in model is to be made the former system requires recalculation of $F_{1}, F_{2}, G_{1}, G_{2}, G_{3}$ in (3.1). The system discussed here requires only a change in the subprogram for computing $\hat{\sigma}_{\alpha}(t)$ from $\lambda_{\beta}(t)$, as in (4.3). Standard methods for solving (4.3) for $\lambda_{1}(t)$ are available and can be the same for all constitutive equations.

The revised system (4.2) becomes even more attractive for use with constitutive equations in which the argument $t-s$ in (2.4) is replaced by a stretch history dependent pseudo-time,

$\xi(r, t)=\int_{s}^{t} \phi\left(\frac{\lambda(r, \alpha)}{\lambda(r, t)}, \alpha\right) \mathrm{d} \alpha$,

as has been proposed in [7]. If the method of Section 3 were to be used, the operation $\partial T_{1} / \partial r$ in (2.2) leads to an equation involving $\partial \xi / \partial r$ which is much more complicated than (3.1). In the revised reduction, the structure of equations (4.2) and the solution procedure is unaffected.

\section{Numerical example}

The membrane material is assumed to be a homogeneous, incompressible viscoelastic fluid which can be modeled as a BKZ fluid [8]. One material which has been used in the membrane inflation experiment and other thin sheet forming studies is polyisobutylene [2]. A specific constitutive equation of the form (2.4) for polyisobutylene which can be used for sheet problems does not appear to be available. For this reason a composite constitutive equation was constructed from several sources $[10,11]$. With respect to principal directions, it has the following form:

$$
\begin{aligned}
& \sigma_{\alpha}(t)=2\left(\lambda_{\alpha}^{2}(t)-\lambda_{3}^{2}(t)\right)\left[1+\frac{4.5\left(1+\lambda_{\beta}^{2}(t)\right)}{I_{1}(t, 0)+I_{2}(t, 0)+3}\right] G(t) \\
& -2 \int_{0}^{t}\left[\frac{\lambda_{\alpha}^{2}(t)}{\lambda_{\alpha}^{2}(s)}-\frac{\lambda_{3}^{2}(t)}{\lambda_{3}^{2}(s)}\right]\left[1+\frac{4.5}{I_{1}(t, s)+I_{2}(t, s)+3}\left(1+\frac{\lambda_{\beta}^{2}(t)}{\lambda_{\beta}^{2}(s)}\right)\right] \times \dot{G}(t-s) \mathrm{d} s,
\end{aligned}
$$

where $\alpha=1, \beta=2$,or $\alpha=2, \beta=1$,

$I_{1}(t, s)=\sum_{i=1}^{3}\left(\frac{\lambda_{i}(t)}{\lambda_{i}(s)}\right)^{2}, \quad I_{2}(t, s)=\sum_{i=1}^{3}\left(\frac{\lambda_{i}(s)}{\lambda_{i}(t)}\right)^{2}$,

and $G(t) \rightarrow 0$ as $t \rightarrow \infty$.

This constitutive equation is a simplified version of one presented by Zapas in [10]. It was developed from biaxial extension experiments and contained 


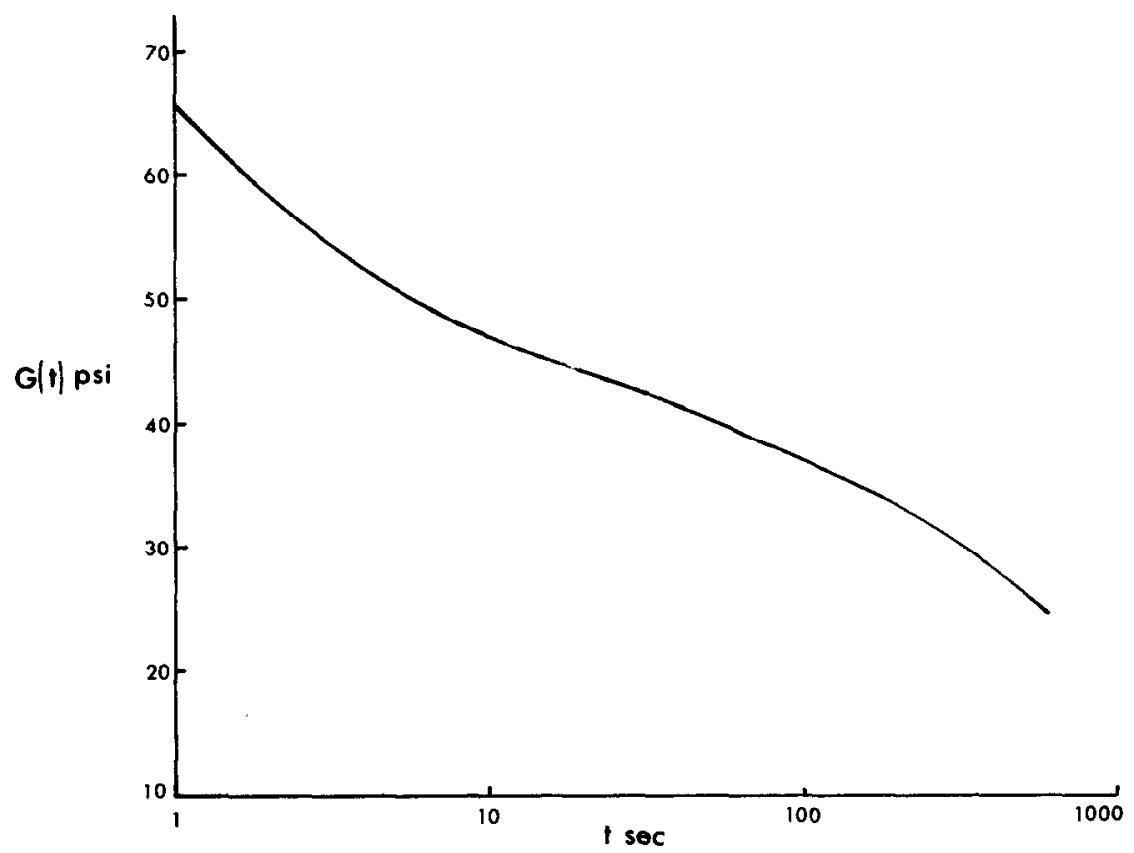

Fig. 2. Assumed form of the relaxation function $G(t)$.

three material dependent functions of time, one of which is $G(t)$. Values for $G(t)$ for polyisobutylene were not given. Instead, $G(t)$ was taken from [11], which considers the response of PIB L-100 at $25^{\circ} \mathrm{C}$ subjected to uniaxial exponential stretch ratio histories. Experimental data was fit to a uniaxial model proposed by Zapas and Craft [12], which is qualitatively similar to that in [10]. Material time functions were determined and presented graphically, see [11], Fig. 21, p. 294. $G(t)$ was chosen to be the same as $\beta(t)+\gamma(t)$ on this graph shown here in Fig. 2. Although (5.1) is admittedly a patchwork constitutive equation, its structure does embody many features which might be anticipated in a correct version. As such, it is certainly useful for the purposes of a numerical example.

For computational purposes, $G(t)$ was fit by a function of $t$ as follows. Values for $G(t)$ at various times between $t=1 \mathrm{sec}$ and $t=600 \mathrm{sec}$ were estimated from its graph. On each of the intervals $1 \leqslant t \leqslant 10,10 \leqslant t \leqslant 100$, $100 \leqslant t \leqslant 600, G(t)$ was represented by a cubic spline. For a typical interval $t_{i} \leqslant t \leqslant t_{j}$, this had the form

$$
\begin{gathered}
G(t)=\bar{G}(\log t)=\left[G_{i}+\left[G_{i}^{\prime} \log \left(t_{i} / t_{j}\right)-2 G_{i}\right] \frac{\log t / t_{i}}{\log t_{i} / t_{j}}\right]\left(\frac{\log t / t_{j}}{\log t_{i} / t_{j}}\right)^{2} \\
+\left[G_{j}+\left[G_{j}^{\prime} \log t_{j} / t_{i}-2 G_{j}\right] \frac{\log t / t_{j}}{\log t_{j} / t_{i}}\right]\left(\frac{\log t / t_{i}}{\log t_{j} / t_{i}}\right)^{2},
\end{gathered}
$$


TABLE 1

\begin{tabular}{lll}
\hline$t_{i}(\mathrm{sec})$ & $G_{i}$ & \multicolumn{1}{c}{$G_{i}^{\prime}$} \\
\hline 1 & 65.437 & -25.960 \\
10 & 47.026 & -9.891 \\
100 & 36.874 & -12.179 \\
600 & 24.720 & -22.605 \\
$\hat{A}=76.712$ & $\hat{B}=-11.274$ \\
$\hat{A}=87.521$ & $\bar{B}=-22.605$ \\
\hline
\end{tabular}

where

$G_{i}=\bar{G}\left(\log t_{i}\right), \quad G_{j}=\bar{G}\left(\log t_{j}\right)$,

$G_{i}^{\prime}=\left.\frac{\mathrm{d} \bar{G}(\log t)}{\mathrm{d}(\log t)}\right|_{t=t_{i}}, \quad G_{j}^{\prime}=\left.\frac{\mathrm{d} \bar{G}(\log t)}{\mathrm{d}(\log t)}\right|_{t=t_{j}}$.

Function values and first derivatives were matched at $t=10,100$. The eight function values and derivatives in this representation were chosen to minimize the square error between the graphical and evaluated values of $G(t)$ at various times.

On the intervals not represented by experimental data, $G(t)$ was extrapolated by,

$0 \leqslant t \leqslant 1: \quad G(t)=\hat{A}+\hat{B} t$,

$600 \leqslant t \leqslant 7442.2: \quad G(t)=\bar{A}+\bar{B} \log t$,

$7442.2 \leqslant t: \quad G(t)=0$,

where $\hat{A}, \hat{B}, \bar{A}, \bar{B}$ were selected to ensure continuity through the first derivative. Values for the various constants appear in Table 1 . It is seen that $G(0)=$ $\hat{A}=76.7$ and $G(t)$ has decayed to zero at $t=7442.2 \mathrm{sec}$.

The selection of times $t_{i}$ at which the solution is to be computed appears to be somewhat arbitrary in solving viscoelasticity problems. For viscoelastic solids, if the pressure reaches a limiting value then a fixed equilibrium state is reached. Time increments can become larger as shape changes become slower. For fluids, even if the pressure is limited, the material will continue to creep. This suggests that time increments cannot become too large. A method of selecting times was used which will be discussed in more detail in a later publication. To select times $t_{i}$, the integral in (5.1) is written as a Stieltjes integral,

$\int_{0}^{t} \bar{B}_{\alpha}(\lambda(t), \lambda(s)) \mathrm{d} G_{t}(s)$

where $G_{t}(s)=G(t-s)$. It is then approximated by

$\int_{0}^{t} \bar{B}_{\alpha}(\lambda(t), \lambda(s)) \mathrm{d} G_{t}(s) \simeq \sum_{k=1}^{n} W_{n k} \bar{B}_{\alpha}\left(\lambda\left(t_{n}\right), \lambda\left(t_{k}\right)\right)\left(G\left(t_{n}-t_{k}\right)-G\left(t_{n}-t_{k-1}\right)\right)$, 


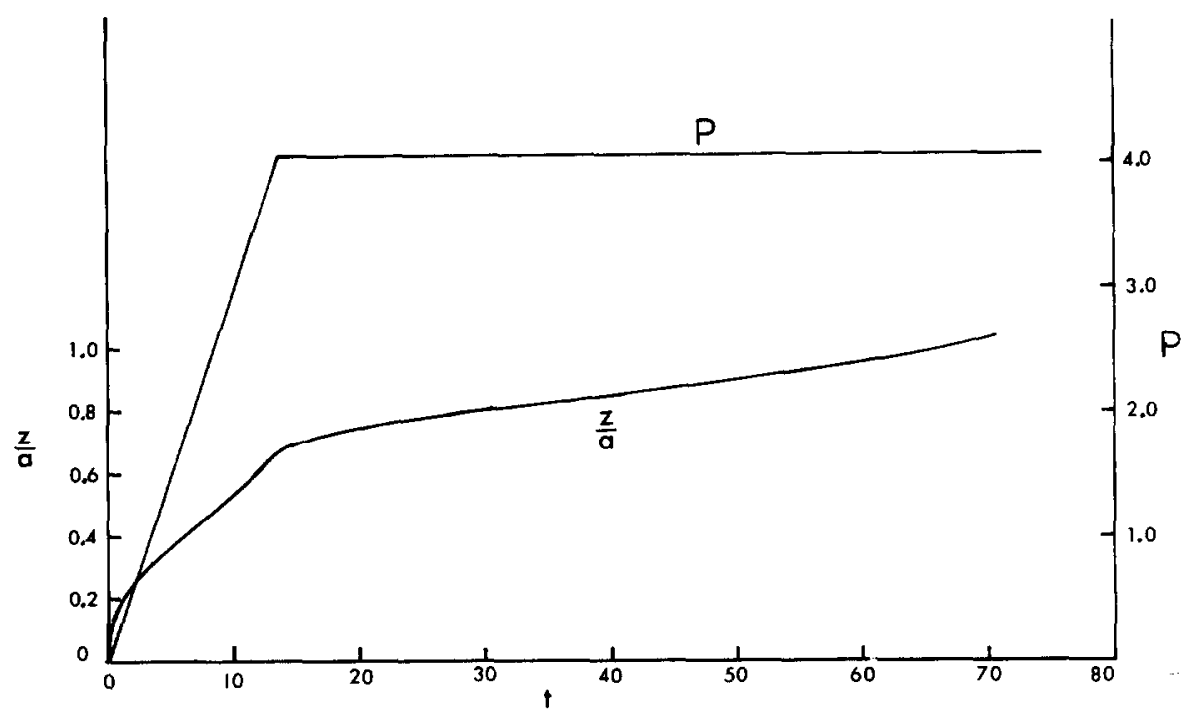

Fig. 3. Dimensionless pressure history $P(t)$ and polar height history $z(0, t) / a$.

using a Simpson's rule for unequal intervals. Times are selected so that the most recent interval is $K$ times the previous one, i.e. $t_{n}$ satisfies

$\left[G(0)-G\left(t_{n}-t_{n-1}\right)\right]=K\left[G\left(t_{n}-t_{n-1}\right)-G\left(t_{n}-t_{n-2}\right)\right]$.

Time $t_{2}$ was selected so that the initial increment in $G(t)$ was 0.01 .

Before computing, equations (4.2) were non-dimensionalized by setting $r \rightarrow r / a, \rho \rightarrow \rho / a, z \rightarrow z / a, \hat{\sigma}_{\alpha} \rightarrow \hat{\sigma}_{\alpha} / G(0)$. The equations are unaltered with the exception that $p / h_{0}$ in the last of (4.2) is replaced by $P=(p a) /\left(h_{0} G(0)\right)$. The pressure history used for computation is shown in Fig. 3. It increases linearly until $P=4.07$ at $t=13.6$ and is then held constant. The maximum height history is also shown. It continues to increase even though the pressure is fixed. Deformed profiles at various times are shown in Fig. 4.

No numerical problems arose in connection with the obtaining of the inverse (4.4) during the integration of (4.2). The only issue of a computational nature has to do with the form of the integrand in the constitutive equation (5.1). The stresses are evaluated many times during the iteration associated with obtaining the inverse (4.4) and selecting $A\left(t_{n}\right)$ to satisfy the boundary condition. During each evaluation, it is necessary to sum the integrals from $t_{1}$ to $t_{n}$, which uses a lot of computing time. This could be avoided if integrals were a polynomial in $I_{1}(t, s)$ and $I_{2}(t, s)$. (5.6) could then be written as

$$
\int_{0}^{t} \bar{B}_{\alpha}(\lambda(t), \lambda(s)) \mathrm{d} G_{t}(s)=\sum_{\gamma=1}^{N} L_{\gamma}(\lambda(t)) \int_{0}^{t} M_{\gamma}(\lambda(s)) \mathrm{d} G_{t}(s)
$$




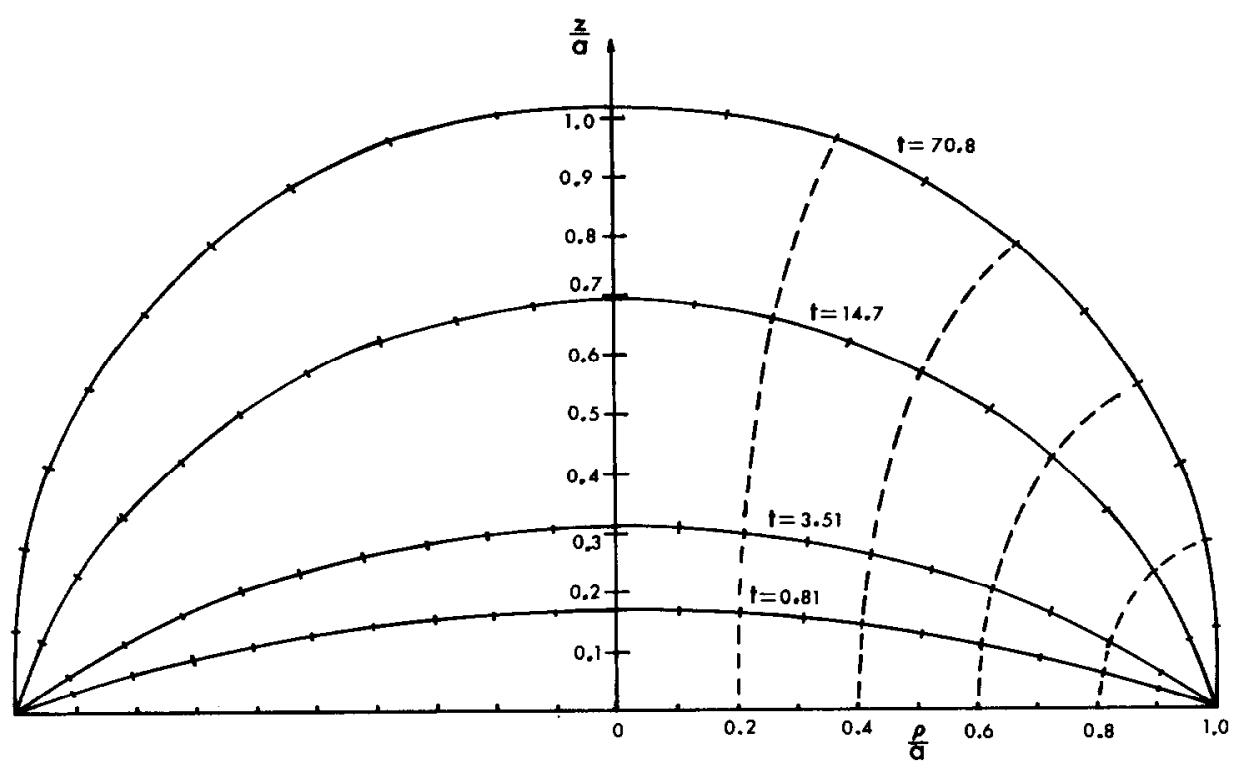

Fig. 4. Deformed profiles of the membrane at various times. Dashed lines denote particle paths.

The integral could then be evaluated only once while iteration involving $\lambda(t)$ proceeds. Since many different choices of functions can fit the same experimental data, it is suggested that constitutive equations be developed with an awareness of computational efficiency.

\section{Acknowledgment}

The author wishes to express his gratitude to Mr. Ramesh Guttalu and Mr. David Zella for their computational assistance and to the National Science Foundation for their support under Grant No. ENG75-17489.

\section{References}

1 A.E. Green and J.E. Adkins, Large Elastic Deformations and Nonlinear Continuum Mechanics, Oxford University Press, 1960.

2 D.D. Joye, G.W. Poehlein and C.D. Denson, Trans. Soc. Rheol., 16 (1972) 421.

3 S.C.-K. Chung and J.F. Stevenson, Rheol. Acta, 14 (1975) 832.

4 A.S. Wineman, Int. J. Solids Struct., 8 (1972) 775.

5 A.S. Wineman, J. Appl. Mech., 39 (1972) 848.

6 A.S. Wineman, Trans. Soc. Rheol., 20 (1976) 203.

7 L.e. Zapas, Deformation and Fracture of High Polymers, Plenum Press, 1974, p. 381.

8 B. Bernstein, E.A. Kearsley and L.J. Zapas, Trans. Soc. Rheol., 7 (1963) 391.

9 B.D. Coleman and W. Noll, Rev. Mod. Phys., 33 (1961) 239.

10 L.J. Zapas, J. Res. NBS, 70A (Physics and Chemistry), (1966) 525.

11 W. Goldberg, B. Bernstein and G. Lianis, Int. J. Non-Linear Mechanics, 4 (1969) 277.

12 L.J. Zapas and T. Craft, J. Res. NBS, 69A (1965) 541. 